\title{
13. CARBON CARBONATE RESULTS, LEG 4
}

\author{
A. C. Pimm, Deep Sea Drilling Project, East Coast Repository \\ Lamont-Doherty Geological Observatory, Palisades, N. Y.
}

\section{INTRODUCTION}

The complete carbon carbonate results from all samples recovered on Leg 4 are given in Table 1. Methods used in the carbon carbonate analysis discussed here are outlined in the shore based laboratory handbook, and are summarized in the appendix to this volume. All of the analyses were made on a LECO 70 Carbon Analyzer.

\section{CALCIUM CARBONATE RESULTS}

\section{Site 23}

Cores 1 through 4 of this hole contain an average of 3.4 per cent carbonate in brown clay. Core 5 contains carbonate values of 20.4 and 9.2 per cent in sand and silt layers, comprised of abundant foraminifera and calcareous nannoplankton; as this site is in 5079 meters of water it is assumed that these carbonate-rich layers in the brown clays are of turbidite origin.

\section{Site 24}

Sediment from Site 24 averages 3 per cent carbonate. The range in carbonate values is from nil in brown clay, to 5.8 per cent in quartz sand layers with calcareous fossils; these are probably of turbidite origin as the water depth here is 5148 meters.

\section{Site 25}

Sediments from Holes 25 and 25A were all foraminiferalnannoplankton chalk oozes averaging 75.5 per cent carbonate.

\section{Site 26}

Sediments here are of terrigenous origin with a low carbonate content averaging 2.0 per cent. They comprise silty clay and sand deposited by turbidity currents.

\section{Site 27}

Brown to green clay in Cores 1 through 4 of Hole 27 contains nil to 2.8 per cent carbonate. In Sections 1 and 2 of Core 5 , the carbonate content is less than 2.3 per cent in similar sediment. In Sections 3 and 4, the carbonate content averages 30 per cent in calcareous clay. The carbonate here is biogenic, but considering the present water depth of 5251 meters, the water was either shallower at the time (Eocene) these sediments were deposited, or the fossils were transported into the area by turbidity currents. The latter possibility is most likely, as in Core 6 the carbonate percentage is again nil in brown-to-green clay.

\section{Site 28}

In Core 2 and Section 1 of Core 3 the carbonate content averages 1 per cent in brown clay. In Core 3, Section 2 and below, the carbonate content increases and averages 27.8 per cent. The sediment here is a calcareous clay containing both calcareous and siliceous fossils. The present water depth here is 5515 meters, and it is not known whether these moderately calcareous sediments were deposited originally (Middle Eocene) in shallower water or are of turbidity current origin. Cores 4 through 9 predominantly comprise calcareous sediments, but brown and green clays in a few places are probably low in carbonate content; no samples were taken for analysis from these cores.

\section{Site 29}

Core 1 through Core 4 , Section 3, averages 25.2 per cent carbonate in nannoplankton clay and marl ooze. From Section 4, Core 4 through Core 8, the carbonate values are normally nil in olive and brown clays, but a few layers containing as much as 55 per cent carbonate in one place are of nannoplankton-foraminiferal marl ooze. Similarly, higher carbonate values of up to 26.5 per cent occur in brown radiolarian oozes from Cores 9 to 18 which generally contain no carbonate. It is not known whether the carbonate content is due to the remnants of calcareous fossils or is of dolomitic origin, but the latter possibility is most likely. For the calculations in Table 1 it has been assumed that all carbonate is in the form of calcium carbonate. However, if dolomite is confirmed then the carbonate values should be recalculated accordingly (see appendix to this volume for details).

In Hole 29B, Core 1 through Core 5, Section 1, consists of olive-brown clay with negligible carbonate values; a few layers contain moderate amounts of carbonate up to 15.9 per cent. In Core 5, Sections 2 to 4 the carbonate content varies from 52.7 to 21.0 per cent in a white marl ooze. From Cores 8 through 10 the carbonate content is usually nil in pale brown radiolarian ooze. 
TABLE 1

Leg 4 Carbonate Results

\begin{tabular}{|c|c|c|c|c|c|c|}
\hline Hole & Core & Section & $\begin{array}{l}\text { Sampled at } \\
(\mathrm{cm})\end{array}$ & $\begin{array}{l}\text { Total Carbon } \\
\text { Per Cent }\end{array}$ & $\begin{array}{l}\text { Organic Carbon } \\
\text { Per Cent }\end{array}$ & $\begin{array}{c}\text { Calcium Carbonate } \\
\text { Per Cent }\end{array}$ \\
\hline 23 & 1 & 1 & 71 & 0.4 & 0.1 & 2.2 \\
\hline 23 & 1 & 2 & 2 & 0.8 & 0.1 & 5.8 \\
\hline 23 & 2 & 1 & 70 & 0.4 & 0.0 & 3.4 \\
\hline 23 & 3 & 2 & 20 & 0.4 & 0.0 & 3.7 \\
\hline 23 & 3 & 3 & 19 & 0.3 & 0.1 & 1.7 \\
\hline 23 & 4 & 1 & 6 & 0.4 & 0.1 & 2.3 \\
\hline 23 & 4 & 3 & 47 & 0.5 & 0.0 & 4.0 \\
\hline 23 & 4 & 4 & 58 & 0.5 & 0.0 & 4.2 \\
\hline 23 & 5 & 1 & 10 & 2.6 & 0.1 & 20.4 \\
\hline 23 & 5 & 1 & 17 & 1.4 & 0.3 & 9.2 \\
\hline 24 & 1 & 1 & 27 & 0.9 & 0.3 & 5.8 \\
\hline 24 & 4 & 2 & 5 & 0.2 & 0.0 & 0.2 \\
\hline 24 & 4 & 3 & 22 & 0.3 & 0.2 & 1.3 \\
\hline 24 & 4 & 4 & 21 & 0.0 & 0.0 & 0.0 \\
\hline $24 \mathrm{~A}$ & 1 & 1 & 24 & 0.6 & 0.0 & 4.9 \\
\hline $24 \mathrm{~A}$ & 2 & 1 & 24 & 0.5 & 0.0 & 4.0 \\
\hline $24 \mathrm{~A}$ & 2 & 1 & 27 & 0.5 & 0.0 & 4.0 \\
\hline $24 \mathrm{~A}$ & 3 & 1 & 135 & 1.2 & 1.2 & 0.0 \\
\hline $24 \mathrm{~A}$ & 4 & 2 & 140 & 1.2 & 0.8 & 2.8 \\
\hline 25 & 1 & 1 & 25 & 7.7 & 0.1 & 63.3 \\
\hline 25 & 1 & 2 & 30 & 8.2 & 0.1 & 67.6 \\
\hline 25 & 1 & 4 & 110 & 8.6 & 0.2 & 70.1 \\
\hline 25 & 1 & 6 & 68 & 9.0 & 0.1 & 74.4 \\
\hline 25 & 2 & 2 & 30 & 9.6 & 0.1 & 78.8 \\
\hline 25 & 2 & 3 & 70 & 8.6 & 0.1 & 70.1 \\
\hline 25 & 3 & 1 & 125 & 9.0 & 0.1 & 74.6 \\
\hline 25 & 3 & 2 & 38 & 9.9 & 0.0 & 82.0 \\
\hline 25 & 3 & 3 & 75 & 9.8 & 0.0 & 81.2 \\
\hline 25 & 3 & 4 & 75 & 9.5 & 0.1 & 78.6 \\
\hline 25 & 3 & 5 & 75 & 9.6 & 0.0 & 79.4 \\
\hline 25 & 3 & 6 & 75 & 10.0 & 0.0 & 82.7 \\
\hline 25 & 4 & 1 & 33 & 8.9 & 0.1 & 73.7 \\
\hline 25 & 4 & 2 & 75 & 10.2 & 0.1 & 84.6 \\
\hline 25 & 4 & 3 & 75 & 9.6 & 0.1 & 79.5 \\
\hline 25 & 4 & 4 & 76 & 9.0 & 0.8 & 68.0 \\
\hline $25 \mathrm{~A}$ & 1 & 2 & 75 & 9.1 & 0.1 & 75.5 \\
\hline 26 & 1 & 2 & 24 & 0.8 & 0.5 & 2.2 \\
\hline 26 & 1 & 3 & 6 & 0.7 & 0.6 & 1.0 \\
\hline
\end{tabular}


TABLE 1 - Continued

\begin{tabular}{|c|c|c|c|c|c|c|}
\hline Hole & Core & Section & $\begin{array}{l}\text { Sampled at } \\
(\mathrm{cm})\end{array}$ & $\begin{array}{l}\text { Total Carbon } \\
\text { Per Cent }\end{array}$ & $\begin{array}{l}\text { Organic Carbon } \\
\text { Per Cent }\end{array}$ & $\begin{array}{c}\text { Calcium Carbonate } \\
\text { Per Cent }\end{array}$ \\
\hline 26 & 2 & 1 & 143 & 0.8 & 0.5 & 2.9 \\
\hline 26 & 2 & 2 & 20 & 0.7 & 0.4 & 2.3 \\
\hline 26 & 3 & 2 & 10 & 0.9 & 0.7 & 2.2 \\
\hline 26 & 4 & 1 & 25 & 1.1 & 0.5 & 4.8 \\
\hline 26 & 5 & 1 & 27 & 0.8 & 0.5 & 2.2 \\
\hline 26 & 5 & 2 & 22 & 0.5 & 0.4 & 0.9 \\
\hline 26 & 5 & 3 & 2 & 0.6 & 0.5 & 0.2 \\
\hline 26 & 5 & 4 & 10 & 0.6 & 0.4 & 1.5 \\
\hline 27 & 1 & 1 & 17 & 0.2 & 0.0 & 1.0 \\
\hline 27 & 1 & 2 & 50 & 0.2 & 0.1 & 1.0 \\
\hline 27 & 1 & 6 & 67 & 0.1 & 0.0 & 0.8 \\
\hline 27 & 2 & 1 & 80 & 0.5 & 0.0 & 3.8 \\
\hline 27 & 2 & 2 & 30 & 0.0 & 0.0 & 0.0 \\
\hline 27 & 2 & 3 & 14 & 0.0 & 0.0 & 0.0 \\
\hline 27 & 3 & 1 & 40 & 0.3 & 0.1 & 1.8 \\
\hline 27 & 3 & 2 & 10 & 0.2 & 0.1 & 0.6 \\
\hline 27 & 4 & 1 & 31 & 1.2 & 0.8 & 3.7 \\
\hline 27 & 4 & 2 & 20 & 0.5 & 0.4 & 1.8 \\
\hline 27 & 4 & 3 & 2 & 0.3 & 0.1 & 1.5 \\
\hline 27 & 5 & 1 & 25 & 0.6 & 0.5 & 0.7 \\
\hline 27 & 5 & 2 & 20 & 0.9 & 0.6 & 2.3 \\
\hline 27 & 5 & 3 & 20 & 4.1 & 0.4 & 31.3 \\
\hline 27 & 5 & 4 & 23 & 4.0 & 0.3 & 30.7 \\
\hline 27 & 6 & 3 & 7 & 0.0 & 0.0 & 0.0 \\
\hline $27 \mathrm{~A}$ & 1 & 1 & 72 & 0.0 & 0.0 & 0.0 \\
\hline $27 \mathrm{~A}$ & 1 & 4 & 60 & 0.1 & 0.1 & 0.0 \\
\hline $27 \mathrm{~A}$ & 1 & 5 & 39 & 0.2 & 0.1 & 0.2 \\
\hline $27 \mathrm{~A}$ & 1 & 6 & 10 & 0.1 & 0.0 & 0.8 \\
\hline $27 \mathrm{~A}$ & 2 & 1 & 50 & 0.0 & 0.0 & 0.0 \\
\hline $27 \mathrm{~A}$ & 2 & 3 & 36 & 0.1 & 0.0 & 0.3 \\
\hline $27 \mathrm{~A}$ & 3 & 1 & 112 & 0.0 & 0.0 & 0.0 \\
\hline $27 \mathrm{~A}$ & 3 & 3 & 20 & 0.0 & 0.0 & 0.0 \\
\hline $27 \mathrm{~A}$ & 4 & 1 & 27 & 0.0 & 0.0 & 0.0 \\
\hline $27 \mathrm{~A}$ & 4 & 2 & 20 & 0.0 & 0.0 & 0.0 \\
\hline $27 \mathrm{~A}$ & 4 & 3 & 32 & 0.0 & 0.0 & 0.0 \\
\hline $27 \mathrm{~A}$ & 4 & 4 & 19 & 0.2 & 0.2 & 0.0 \\
\hline $27 \mathrm{~A}$ & 4 & 4 & 76 & 0.0 & 0.0 & 0.0 \\
\hline $27 \mathrm{~A}$ & 4 & 5 & 64 & 0.2 & 0.2 & 0.0 \\
\hline $27 \mathrm{~A}$ & 4 & 6 & 24 & 0.0 & 0.0 & 0.0 \\
\hline
\end{tabular}


TABLE 1 - Continued

\begin{tabular}{|c|c|c|c|c|c|c|}
\hline Hole & Core & Section & $\begin{array}{l}\text { Sampled at } \\
(\mathrm{cm})\end{array}$ & $\begin{array}{l}\text { Total Carbon } \\
\text { Per Cent }\end{array}$ & $\begin{array}{l}\text { Organic Carbon } \\
\text { Per Cent }\end{array}$ & $\begin{array}{c}\text { Calcium Carbonate } \\
\text { Per Cent }\end{array}$ \\
\hline $27 \mathrm{~A}$ & 5 & 1 & 20 & 0.1 & 0.0 & 0.6 \\
\hline $27 \mathrm{~A}$ & 5 & 2 & 19 & 0.0 & 0.0 & 0.0 \\
\hline $27 \mathrm{~A}$ & 5 & 3 & 18 & 0.1 & 0.0 & 0.5 \\
\hline $27 \mathrm{~A}$ & 5 & 4 & 62 & 0.1 & 0.1 & 0.0 \\
\hline $27 \mathrm{~A}$ & 5 & 5 & 60 & 0.1 & 0.1 & 0.0 \\
\hline 28 & 2 & 2 & 130 & 0.1 & 0.0 & 0.3 \\
\hline 28 & 2 & 3 & 10 & 0.0 & 0.0 & 0.0 \\
\hline 28 & 2 & 4 & 15 & 0.5 & 0.0 & 3.8 \\
\hline 28 & 3 & 1 & 35 & 0.0 & 0.0 & 0.0 \\
\hline 28 & 3 & 2 & 32 & 3.7 & 0.5 & 26.4 \\
\hline 28 & 3 & 3 & 25 & 3.4 & 0.5 & 24.8 \\
\hline 28 & 3 & 4 & 7 & 3.3 & 0.5 & 24.0 \\
\hline 28 & 3 & 5 & 6 & 3.9 & 0.0 & 32.0 \\
\hline 28 & 3 & 6 & 12 & 3.9 & 0.0 & 32.0 \\
\hline 29 & 1 & 1 & 40 & 4.7 & 0.1 & 38.5 \\
\hline 29 & 1 & 2 & 40 & 2.5 & 1.0 & 12.8 \\
\hline 29 & 1 & 3 & 18 & 2.5 & 0.2 & 19.5 \\
\hline 29 & 2 & 1 & 34 & 3.1 & 0.0 & 26.1 \\
\hline 29 & 2 & 2 & 37 & 5.0 & 3.1 & 41.1 \\
\hline 29 & 2 & 3 & 20 & 3.2 & 0.0 & 26.4 \\
\hline 29 & 3 & 1 & 27 & 2.7 & 0.1 & 22.2 \\
\hline 29 & 4 & 1 & 90 & 2.7 & 0.1 & 21.9 \\
\hline 29 & 4 & 2 & 20 & 2.9 & 0.0 & 24.0 \\
\hline 29 & 4 & 3 & 39 & 2.4 & 0.1 & 19.3 \\
\hline 29 & 4 & 4 & 40 & 1.0 & 0.3 & 0.6 \\
\hline 29 & 5 & 1 & 81 & 0.0 & 0.0 & 0.0 \\
\hline 29 & 6 & 1 & 97 & 0.0 & 0.0 & 0.0 \\
\hline 29 & 7 & 1 & 131 & 6.8 & 0.2 & 55.1 \\
\hline 29 & 8 & 1 & 113 & 0.0 & 0.0 & 0.0 \\
\hline 29 & 9 & 1 & 30 & 2.3 & 0.0 & 19.3 \\
\hline 29 & 9 & 2 & 42 & 0.0 & 0.0 & 0.0 \\
\hline 29 & 9 & 3 & 42 & 1.8 & 0.0 & 15.0 \\
\hline 29 & 9 & 4 & 23 & 0.8 & 0.0 & 6.3 \\
\hline 29 & 9 & 5 & 31 & 0.0 & 0.0 & 0.0 \\
\hline 29 & 9 & 6 & 24 & 0.0 & 0.0 & 0.0 \\
\hline 29 & 10 & 1 & 70 & 0.0 & 0.0 & 0.0 \\
\hline 29 & 10 & 2 & 0 & 0.0 & 0.0 & 0.0 \\
\hline 29 & 10 & 3 & 21 & 0.0 & 0.0 & 0.0 \\
\hline 29 & 10 & 4 & 17 & 0.0 & 0.0 & 0.0 \\
\hline
\end{tabular}


TABLE 1 - Continued

\begin{tabular}{|c|c|c|c|c|c|c|}
\hline Hole & Core & Section & $\begin{array}{l}\text { Sampled at } \\
\quad(\mathrm{cm})\end{array}$ & $\begin{array}{l}\text { Total Carbon } \\
\text { Per Cent }\end{array}$ & $\begin{array}{l}\text { Organic Carbon } \\
\text { Per Cent }\end{array}$ & $\begin{array}{c}\text { Calcium Carbonate } \\
\text { Per Cent }\end{array}$ \\
\hline 29 & 10 & 5 & 40 & 0.0 & 0.0 & 0.0 \\
\hline 29 & 11 & 1 & 99 & 0.0 & 0.0 & 0.0 \\
\hline 29 & 12 & 1 & 13 & 0.3 & 0.1 & 1.8 \\
\hline 29 & 12 & 2 & 23 & 0.0 & 0.0 & 0.0 \\
\hline 29 & 12 & 3 & 32 & 0.9 & 0.1 & 6.9 \\
\hline 29 & 12 & 4 & 126 & 1.6 & 0.2 & 11.9 \\
\hline 29 & 12 & 5 & 37 & 1.8 & 0.1 & 14.3 \\
\hline 29 & 12 & 6 & 25 & 2.1 & 0.2 & 15.8 \\
\hline 29 & 14 & 1 & 20 & 0.2 & 0.2 & 0.0 \\
\hline 29 & 14 & 2 & 21 & 0.0 & 0.0 & 0.0 \\
\hline 29 & 14 & 3 & 20 & 0.0 & 0.0 & 0.0 \\
\hline 29 & 14 & 4 & 19 & 0.0 & 0.0 & 0.0 \\
\hline 29 & 14 & 5 & 20 & 0.0 & 0.0 & 0.0 \\
\hline 29 & 14 & 6 & 20 & 0.4 & 0.2 & 1.7 \\
\hline 29 & 15 & 1 & 20 & 1.0 & 0.1 & 8.1 \\
\hline 29 & 15 & 2 & 20 & 1.9 & 0.0 & 15.7 \\
\hline 29 & 15 & 3 & 20 & 0.6 & 0.0 & 5.1 \\
\hline 29 & 15 & 4 & 20 & 1.0 & 0.0 & 8.0 \\
\hline 29 & 15 & 5 & 29 & 0.3 & 0.0 & 2.4 \\
\hline 29 & 15 & 6 & 20 & 1.1 & 0.0 & 9.4 \\
\hline 29 & 16 & 1 & 60 & 2.4 & 0.0 & 19.9 \\
\hline 29 & 16 & 2 & 20 & 3.2 & 0.0 & 26.5 \\
\hline 29 & 16 & 3 & 20 & 1.1 & 0.4 & 5.4 \\
\hline 29 & 16 & 4 & 20 & 1.4 & 0.0 & 12.0 \\
\hline 29 & 16 & 5 & 21 & 1.3 & 0.0 & 11.0 \\
\hline 29 & 16 & 6 & 20 & 1.3 & 0.0 & 10.4 \\
\hline 29 & 17 & 1 & 10 & 0.0 & 0.0 & 0.0 \\
\hline 29 & 17 & 2 & 20 & 0.2 & 0.0 & 2.0 \\
\hline 29 & 17 & 3 & 20 & 0.1 & 0.0 & 0.5 \\
\hline 29 & 17 & 4 & 20 & 0.1 & 0.0 & 0.5 \\
\hline 29 & 17 & 5 & 20 & 0.0 & 0.0 & 0.0 \\
\hline 29 & 17 & 6 & 20 & 0.0 & 0.0 & 0.0 \\
\hline 29 & 18 & 2 & 30 & 0.2 & 0.0 & 1.4 \\
\hline $29 \mathrm{~A}$ & 4 & 1 & 23 & 0.0 & 0.0 & 0.0 \\
\hline $29 \mathrm{~A}$ & 5 & 1 & 54 & 0.0 & 0.0 & 0.0 \\
\hline $29 B$ & 1 & 1 & 30 & 0.0 & 0.0 & 0.0 \\
\hline $29 B$ & 1 & 4 & 110 & 0.0 & 0.0 & 0.0 \\
\hline $29 \mathrm{~B}$ & 1 & 5 & 70 & 0.0 & 0.0 & 0.0 \\
\hline 29B & 1 & 6 & 130 & 0.0 & 0.0 & 0.0 \\
\hline
\end{tabular}


TABLE 1 - Continued

\begin{tabular}{|c|c|c|c|c|c|c|}
\hline Hole & Core & Section & $\begin{array}{l}\text { Sampled at } \\
(\mathrm{cm})\end{array}$ & $\begin{array}{l}\text { Total Carbon } \\
\text { Per Cent }\end{array}$ & $\begin{array}{l}\text { Organic Carbon } \\
\text { Per Cent }\end{array}$ & $\begin{array}{c}\text { Calcium Carbonate } \\
\text { Per Cent }\end{array}$ \\
\hline $29 B$ & 2 & 2 & 7 & 0.0 & 0.0 & 0.0 \\
\hline $29 B$ & 2 & 3 & 17 & 1.3 & 0.1 & 10.2 \\
\hline $29 B$ & 2 & 4 & 17 & 0.0 & 0.0 & 0.0 \\
\hline $29 \mathrm{~B}$ & 3 & 1 & 20 & 1.9 & 0.0 & 15.9 \\
\hline $29 B$ & 3 & 2 & 15 & 0.0 & 0.0 & 0.0 \\
\hline $29 B$ & 4 & 1 & 14 & 0.1 & 0.1 & 0.0 \\
\hline $29 \mathrm{~B}$ & 4 & 2 & 15 & 0.0 & 0.0 & 0.0 \\
\hline $29 \mathrm{~B}$ & 4 & 3 & 20 & 0.0 & 0.0 & 0.0 \\
\hline $29 B$ & 4 & 4 & 16 & 0.0 & 0.0 & 0.0 \\
\hline $29 \mathrm{~B}$ & 5 & 1 & 35 & 0.2 & 0.0 & 1.1 \\
\hline $29 B$ & 5 & 2 & 11 & 6.4 & 0.1 & 52.7 \\
\hline $29 B$ & 5 & 3 & 25 & 5.1 & 0.0 & 42.2 \\
\hline $29 \mathrm{~B}$ & 5 & 4 & 34 & 2.5 & 0.0 & 21.0 \\
\hline $29 B$ & 8 & 1 & 10 & 0.0 & 0.0 & 0.0 \\
\hline $29 \mathrm{~B}$ & 8 & 2 & 14 & 0.0 & 0.0 & 0.0 \\
\hline $29 B$ & 8 & 3 & 21 & 0.1 & 0.1 & 0.0 \\
\hline $29 \mathrm{~B}$ & 8 & 4 & 13 & 0.0 & 0.0 & 0.0 \\
\hline $29 B$ & 8 & 5 & 23 & 0.0 & 0.0 & 0.0 \\
\hline $29 B$ & 8 & 6 & 10 & 0.0 & 0.0 & 0.0 \\
\hline $29 \mathrm{~B}$ & 9 & 1 & 10 & 1.0 & 0.0 & 8.5 \\
\hline $29 \mathrm{~B}$ & 9 & 2 & 9 & 0.8 & 0.0 & 6.8 \\
\hline $29 B$ & 9 & 3 & 15 & 0.1 & 0.0 & 0.8 \\
\hline $29 B$ & 9 & 4 & 13 & 0.3 & 0.0 & 2.1 \\
\hline $29 B$ & 9 & 5 & 10 & 0.0 & 0.0 & 0.0 \\
\hline $29 \mathrm{~B}$ & 9 & 6 & 11 & 0.0 & 0.0 & 0.0 \\
\hline $29 B$ & 10 & 2 & 0 & 0.0 & 0.0 & 0.0 \\
\hline $29 B$ & 10 & 3 & 15 & 0.2 & 0.0 & 0.1 \\
\hline $29 \mathrm{~B}$ & 10 & 4 & 15 & 0.0 & 0.0 & 0.0 \\
\hline $29 B$ & 10 & 5 & 38 & 0.0 & 0.0 & 0.0 \\
\hline 30 & 1 & 1 & 91 & 2.2 & 0.5 & 14.2 \\
\hline 30 & 1 & 5 & 21 & 2.3 & 0.4 & 15.2 \\
\hline 30 & 2 & 2 & 106 & 1.8 & 0.2 & 13.4 \\
\hline 30 & 2 & 3 & 26 & 2.8 & 0.3 & 21.1 \\
\hline 30 & 2 & 4 & 112 & 1.9 & 0.4 & 12.5 \\
\hline 30 & 3 & 2 & 20 & 1.0 & 0.5 & 4.3 \\
\hline 30 & 3 & 3 & 70 & 1.3 & 0.5 & 6.3 \\
\hline 30 & 3 & 4 & 10 & 0.5 & 0.4 & 1.2 \\
\hline 30 & 4 & 2 & 17 & 1.1 & 0.5 & 5.3 \\
\hline 30 & 4 & 2 & 60 & 4.5 & 0.5 & 33.9 \\
\hline
\end{tabular}


TABLE 1 - Continued

\begin{tabular}{|c|c|c|c|c|c|c|}
\hline Hole & Core & Section & $\begin{array}{l}\text { Sampled at } \\
\quad(\mathrm{cm})\end{array}$ & $\begin{array}{c}\text { Total Carbon } \\
\text { Per Cent }\end{array}$ & $\begin{array}{l}\text { Organic Carbon } \\
\text { Per Cent }\end{array}$ & $\begin{array}{c}\text { Calcium Carbonate } \\
\text { Per Cent }\end{array}$ \\
\hline 30 & 5 & 1 & 56 & 2.2 & 0.5 & 14.5 \\
\hline 30 & 5 & 2 & 10 & 2.3 & 0.5 & 15.1 \\
\hline 30 & 6 & 1 & 20 & 2.1 & 0.5 & 12.9 \\
\hline 30 & 6 & 2 & 90 & 1.5 & 0.5 & 8.3 \\
\hline 30 & 6 & 3 & 26 & 1.3 & 0.3 & 8.3 \\
\hline 30 & 7 & 1 & 56 & 1.3 & 0.7 & 5.0 \\
\hline 30 & 8 & 1 & 16 & 3.2 & 0.4 & 23.1 \\
\hline 30 & 9 & 2 & 23 & 2.6 & 0.4 & 18.7 \\
\hline 30 & 10 & 1 & 26 & 2.1 & 0.2 & 15.7 \\
\hline 30 & 10 & 2 & 68 & 3.2 & 0.3 & 24.7 \\
\hline 30 & 10 & 3 & 54 & 2.1 & 0.4 & 13.7 \\
\hline 30 & 11 & 2 & 62 & 6.3 & 0.5 & 48.7 \\
\hline 30 & 12 & 3 & 53 & 5.8 & 0.6 & 43.4 \\
\hline 30 & 12 & 4 & 6 & 6.9 & 0.7 & 51.7 \\
\hline 30 & 12 & 5 & 5 & 5.6 & 0.6 & 42.1 \\
\hline 30 & 13 & 1 & 148 & 6.3 & 0.6 & 47.7 \\
\hline 30 & 13 & 3 & 7 & 6.9 & 0.5 & 53.4 \\
\hline 30 & 14 & 1 & 143 & 7.2 & 0.3 & 57.0 \\
\hline 30 & 15 & 2 & 10 & 6.0 & 0.3 & 47.3 \\
\hline 30 & 15 & 4 & 8 & 7.1 & 0.4 & 55.9 \\
\hline 30 & 16 & 1 & 137 & 8.4 & 0.3 & 67.5 \\
\hline 31 & 1 & 1 & 29 & 7.1 & 0.2 & 57.1 \\
\hline 31 & 1 & 2 & 130 & 5.4 & 0.2 & 43.2 \\
\hline 31 & 1 & 4 & 20 & 5.2 & 0.1 & 43.0 \\
\hline 31 & 1 & 5 & 49 & 6.4 & 0.1 & 52.6 \\
\hline 31 & 3 & 1 & 7 & 6.4 & 0.1 & 52.6 \\
\hline 31 & 3 & 2 & 23 & 5.8 & 0.2 & 47.2 \\
\hline 31 & 3 & 3 & 43 & 6.1 & 0.2 & 49.4 \\
\hline 31 & 3 & 5 & 41 & 5.3 & 0.2 & 41.2 \\
\hline 31 & 3 & 6 & 26 & 5.7 & 0.2 & 46.2 \\
\hline 31 & 5 & 1 & 51 & 6.0 & 0.2 & 48.5 \\
\hline 31 & 6 & 1 & 40 & 6.1 & 0.1 & 49.3 \\
\hline 31 & 6 & 2 & 16 & 5.8 & 0.2 & 46.8 \\
\hline 31 & 7 & 1 & 123 & 2.3 & 0.3 & 16.8 \\
\hline 31 & 8 & 1 & 54 & 6.2 & 0.1 & 51.2 \\
\hline 31 & 8 & 2 & 15 & 8.1 & 0.1 & 66.6 \\
\hline 31 & 9 & 2 & 4 & 7.4 & 0.1 & 61.6 \\
\hline 31 & 10 & 1 & 148 & 8.8 & 0.1 & 72.8 \\
\hline 31 & 10 & 2 & 143 & 9.2 & 0.0 & 76.1 \\
\hline 31 & 10 & 4 & 145 & 7.8 & 0.1 & 64.1 \\
\hline 31 & 10 & 5 & 145 & 8.8 & 0.1 & 72.6 \\
\hline
\end{tabular}


The present water depth at Site 29 is 4247 meters, which is close to the carbonate compensation depth in these latitudes. It is not known how marls can occur within a non-carbonate sequence in Hole 29B, unless they are of turbidity current origin, or there have been fluctuations in water depth in this area which may also have been close to the carbonate compensation depth in the Lower Miocene.

\section{Site 30}

Mainly gray calcareous clay occurs in Cores 1 through 10; the carbonate content is due to the presence of calcareous fossils, and averages vary from 3.9 to 19.2 per cent for each core. From Cores 11 through 16 the carbonate content shows a marked increase to an average of 51.5 per cent for all cores. This increase in carbonate is due to a corresponding increase in the number of pelagic foraminifera in these marl oozes.

\section{Site 31}

This hole penetrated nannoplankton-foraminifera marl ooze with an average carbonate content of 45.7 per cent in Cores 1 through 7. From Cores 8 through 10 the carbonate percentage increases to an average of 66.4 per cent in a white nannoplankton chalk ooze.

\section{ORGANIC CARBON RESULTS}

It can be seen from Table 2 that most of the holes penetrated on Leg 4 were very low in organic carbon, either containing none or less than 0.2 per cent. Two holes, however, showed consistently higher values. Hole 26 maintained an average of 0.5 per cent organic carbon; here the sediments are sandy turbidites containing abundant plant remains. Hole 30 had an average organic carbon content of 0.4 per cent; the sediments here were marl or chalk oozes with a high glauconite content.

TABLE 2

Summary of Organic Carbon Results from Leg 4

\begin{tabular}{|c|c|c|}
\hline Hole & $\begin{array}{l}\text { Organic } \\
\text { Carbon } \\
\text { Per Cent }\end{array}$ & Comment \\
\hline 23 & $0.0-0.1$ & \\
\hline $24,24 \mathrm{~A}$ & $0.0-0.3$ & $\begin{array}{l}\text { Two high readings of } 0.8 \\
\text { and } 1.2 \% \text {. }\end{array}$ \\
\hline $25,25 \mathrm{~A}$ & $0.0-0.2$ & $\begin{array}{l}\text { One high reading of } \\
0.8 \% \text {. }\end{array}$ \\
\hline 26 & 0.5 average & \\
\hline 27 & $0.0-0.1$ & $\begin{array}{l}\text { In Cores } 4 \text { and } 5 \text {, aver- } \\
\text { ages } 0.4 \% \text {. }\end{array}$ \\
\hline 28 & 0.0 & $\begin{array}{l}\text { In Core } 3 \text { Sections } 2-4 \text {, } \\
\text { average } 0.5 \% \text {. }\end{array}$ \\
\hline 29 & $0.0-0.2$ & $\begin{array}{l}\text { High reading of } 1.0 \% \text { in } \\
\text { Core } 1 .\end{array}$ \\
\hline 29A, 29B & $0.0-0.1$ & \\
\hline 30 & 0.4 average & \\
\hline 31 & 0.1 average & \\
\hline
\end{tabular}

